\title{
Optical Coherence Tomography Accurately Measures Corneal Power Change From Laser Refractive Surgery
}

\author{
Ryan P. McNabb, PhD $^{1,2,{ }^{*}}$, Sina Farsiu, $\mathrm{PhD}^{1,2}$, Sandra S. Stinnett, $\mathrm{DPH}^{2,3}$, Joseph A. Izatt, \\ $\mathrm{PhD}^{1,2}$, and Anthony N. Kuo, MD ${ }^{2}$ \\ ${ }_{1}^{1}$ Department of Biomedical Engineering, Duke University, Durham, NC 27708, USA \\ ${ }^{2}$ Department of Ophthalmology, Duke University, Durham, NC 27710, USA \\ ${ }^{3}$ Department of Biostatistics and Bioinformatics, Duke University, Durham, NC 27710, USA
}

\begin{abstract}
Purpose-To determine the ability of motion corrected optical coherence tomography (OCT) to measure the corneal refractive power change due to laser in situ keratomileusis (LASIK).

Design-Evaluation of a diagnostic test or technology in a cohort.

Subjects-70 eyes from 37 subjects undergoing LASIK were measured preoperatively. 39 eyes from 22 subjects were measured postoperatively and completed the study.

Methods-Consecutive patients undergoing LASIK at the Duke Eye Center who consented to participate were imaged with Placido-ring topography, Scheimpflug photography and OCT on the day of their surgery. Patients were then reimaged with the same imaging systems at the postoperative month 3 visit. Change in pre- to post-operative corneal refractive power as measured by each of the imaging modalities was compared to the pre- to post-operative change in manifest refraction using t-test with generalized estimating equations.
\end{abstract}

Main Outcome Measures-Corneal refractive power change due to LASIK as measured by Placido-ring topography, Scheimpflug Photography, and OCT compared to the manifest refraction change vertexed to the corneal plane. The change in manifest refraction should correspond to the

\footnotetext{
(C) 2014 by the American Academy of Ophthalmology. All rights reserved.

*To whom correspondence should be addressed: ryan.mcnabb@duke.edu. Reprint Address: Ryan P. McNabb, DUMC 3802, 2351 Erwin Road, Durham, NC 27710

Conflict of Interest: Through Duke University, JAI, RPM, and ANK, are co-authors on U.S. Patent 8,403,481 describing distributed scanning optical coherence tomography. Portions of this research made use of products, components, and/or software manufactured by Bioptigen, Inc. Duke University has equity and intellectual property interests (including royalties) with Bioptigen. JAI has corporate, equity, and intellectual property interests (including royalties) with Bioptigen.

Author contributions: RPM and JAI invented and designed the modifications to the OCT system. RPM, ANK, and JAI designed the study. RPM and ANK conducted the study. RPM, SF, SSS, ANK, and JAI analyzed the data. RPM, SF, SSS, ANK, and JAI drafted and revised the manuscript.

Data and materials availability: N/A

Publisher's Disclaimer: This is a PDF file of an unedited manuscript that has been accepted for publication. As a service to our customers we are providing this early version of the manuscript. The manuscript will undergo copyediting, typesetting, and review of the resulting proof before it is published in its final citable form. Please note that during the production process errors may be discovered which could affect the content, and all legal disclaimers that apply to the journal pertain.
} 
change in the corneal refractive power from LASIK and was considered the reference measurement.

Results-In 22 returning post-LASIK individuals (39 eyes), we found no significant difference between the clinically measured pre to post LASIK change in manifest refraction and both Scheimpflug photography $(p=0.714)$ and OCT $(p=0.216)$. In contrast, keratometry values from Placido-ring topography were found to be significantly different from the measured refractive change $(p<0.001)$. Additionally, of the three imaging modalities, OCT recorded the smallest mean absolute difference from the reference measurement with the least amount of variability.

Conclusion-Motion corrected OCT more accurately measures the change in corneal refractive power due to laser refractive surgery than currently available clinical devices. By offering accurate corneal refractive power measurements in normal and surgically modified subjects, OCT offers a compelling alternative to current clinical devices for determining corneal refractive power.

The ability to accurately measure corneal optical power after LASIK and other popular laser refractive surgeries is an important diagnostic challenge. ${ }^{1}$ Over 12 million individuals - and counting - have already undergone laser refractive surgery. As these individuals age, they will naturally develop visually significant cataracts, an ailment which will affect 1 out of every 2 individuals by age 80 in the United States. ${ }^{2}$ While cataract surgery is one of the most commonly performed and most successful surgical procedures in modern medicine, an important requisite is the ability to accurately measure the optical properties of the eye before surgery. Current clinical instruments have difficulty measuring corneal refractive power in individuals who have undergone laser refractive surgery, which has resulted in ambiguities in surgical planning and unpredictable, undesired refractive cataract outcomes for these patients. ${ }^{3}$

Current clinical instruments to measure corneal power - keratometers and Placido-ring topographers - measure the shape of the front surface of the cornea. From only this front surface measurement, assumptions are used to determine the optical power of the whole cornea. ${ }^{4,5}$ Laser refractive surgery is effective because it alters the front surface curvature of the cornea, thereby changing its optical power. Unfortunately, this undermines the very assumptions used by keratometers to determine corneal power. This has resulted in inaccurate corneal power measurements with undesired visual outcomes after cataract surgery, ${ }^{3}$ and for these individuals, additional surgery with the attendant risks of reoperation may be required to achieve the intended result. This diagnostic dilemma has led to the creation of multiple disparate methods to compensate for the shortfalls in the current diagnostic instruments. ${ }^{6-14}$ There currently remains no established single method to clinically determine the power of the cornea for individuals after laser refractive surgery.

In contrast to keratometry and topography, tomographic imaging techniques such as optical coherence tomography (OCT) are able to volumetrically image the cornea. OCT is based on low coherence optical interferometry and is capable of clinical micrometer scale imaging. ${ }^{15-21}$ Volumetric images of the cornea contain both front and back corneal surfaces and its thickness - the elements needed to determine corneal power. OCT hence offers the promise of improved measurement of corneal power, even in corneas altered by laser refractive surgery. ${ }^{22}$ 
While prior efforts have examined only the post LASIK corneal power measurement with OCT and shown that isolated measurement to be different from standard diagnostics, ${ }^{22}$ we show in this work the first demonstration that OCT accurately and quantitatively measures the actual pre- to post- change in corneal power due to LASIK. Further, we provide the first demonstration of the additional ability to measure the astigmatic change as well. Together, these findings have important implications for the looming cataract surgery outcomes of the large number of individuals who have had or will undergo laser refractive surgery.

\section{Methods}

\section{OCT System Design for Corneal Biometry}

OCT utilizes a flying spot scanning technique to acquire a volume, where each spot corresponds to a single depth profile, or A-scan. The volume is then built from sequentially acquired A-scans. Because each point within a given volume is acquired at a separate, distinct point in time, patient motion during volume acquisition is encoded within the images of the volume. To reduce the effects of patient motion during acquisition, we utilized a technique we previously developed termed distributed scanning OCT (DSOCT). ${ }^{23}$ DSOCT uses a custom scanning protocol applied on a commercial spectral domain OCT instrument that reduces the temporal correlation between adjacent A-scans and allows for motion estimation and removal of patient bulk motion that occurs during volume acquisition. Using DSOCT, we previously found that differences in corneal power measurements between OCT and standard keratometric techniques were reduced over no motion correction in normal eyes, ${ }^{23,} 24$ Figure 1 illustrates the DSOCT technique. Figure $1 \mathrm{~A}$ shows multiple corneal profiles from several meridians in a DSOCT volume. Figure 1B shows the multiple corneal profiles collected along a single meridian. The multiple passes within the meridian are motion compensated and interlaced to generate a critically sampled B-scan as illustrated in Fig. 1C. After recovery of the surface profiles using fully automated segmentation and correcting for optical artifacts, ${ }^{24-26}$ spherical and astigmatic refractive power and corneal topographic maps can be measured (Fig. 1D). ${ }^{23,27}$

In this study, the DSOCT software was implemented on a commercially available spectral domain OCT system ( $\lambda=840 \mathrm{~nm}, \Delta \lambda=49 \mathrm{~nm}$, Bioptigen, Inc.). The OCT system acquisition rate was 10,000 A-scans per second. We used a temporally distributed rosette scan pattern that resulted in a volume that was equivalent to a conventional radial scan pattern consisting of 20 meridians and $500 \mathrm{~A}$-scans per meridian. The reconstruction algorithm was updated from $\mathrm{McNabb}$, et al. ${ }^{23}$ to denoise and directly register the subsampled meridians to improve the speed and accuracy of the reconstruction algorithm. Biometric values were then determined as described in the previous paragraph.

\section{Subject Imaging and Protocol}

All human subject research was approved by the Duke University Medical Center Institutional Review Board and was HIPAA compliant. Prior to imaging, informed consent was received for all subjects after explanation of the possible consequences and nature of the study. All portions of this research followed the tenets of the Declaration of Helsinki. 
The population for this study was drawn from healthy volunteers at the Duke Eye Center undergoing planned LASIK surgery for refraction correction. All surgeries were performed by the same surgeon. On the day of and prior to surgery, each subject received the following protocol for each eye undergoing LASIK surgery: manifest refraction, corneal topography (Atlas 995®; Carl Zeiss Meditec Inc.; Dublin, CA), Scheimpflug photography (Pentacam ${ }^{\circledR}$ V2.73r19; Oculus Optikgeräte GmbH; Wetzlar, Germany), and OCT (Bioptigen, Inc.; Durham, NC) with custom DSOCT scan protocol and post-processing applied as described above. We performed all imaging modalities in triplicate with the mean value used for subsequent analysis. Subjects who returned to the Duke Eye Center for their $3+$ month postoperative evaluation repeated the above protocol. Measurements from the pre-operative evaluation for all subjects were used to compare the accuracy and repeatability of OCT with motion correction to corneal topography and Scheimpflug photography. For subjects where we obtained pre- and postoperative manifest refraction and imaging data, we compared the measured change in spherical equivalent and astigmatic power from each imaging modality to the measured change as evaluated by manifest refraction. Repeatability of the imaging modalities in post-operative subjects was also computed.

\section{Imaging Device Corneal Biometry Measurements}

For corneal topography, we calculated the spherical equivalent power, $\Phi_{\mathrm{K}}$, from the mean value of the reported SimK powers $\Phi_{\mathrm{K} 1}$ (at angle $\angle_{\mathrm{K} 1}$ ) and $\Phi_{\mathrm{K} 2}$ (at angle $\angle_{\mathrm{K} 2}$ ) over a nominal $3 \mathrm{~mm}$ zone. We calculated the astigmatic power, $\Phi_{\mathrm{Cyl}}$, from the formula $\mid \Phi_{\mathrm{K} 1}-$ $\Phi_{\mathrm{K} 2}$ and $\angle_{\mathrm{Cy} 1}$ from $\angle_{\mathrm{K} 1}$ where $\angle_{\mathrm{K} 1}$ is the major axis. We calculated the corneal anterior surface radius of curvature, $r_{a}$, from the formula $337.5 / \Phi_{\mathrm{K}}$ where 337.5 is equal to the keratometric constant, $1000 *\left(n_{k}-n_{a}\right)$. The indices of refraction $n_{k}=1.3375$ and $n_{a}=1.000$ are the keratometric index and the index of refraction for air respectively. 5,28

For Scheimpflug photography, the scanning protocol consisted of 25 radial meridians with a sample spacing of $0.1 \mathrm{~mm}$. We obtained $\Phi_{\mathrm{K}}$ from the reported EKR value for a $3 \mathrm{~mm}$ zone. ${ }^{29}$ We calculated $\Phi_{\mathrm{Cyl}}$ from the formula $\left|\Phi_{\mathrm{K} 1}-\Phi_{\mathrm{K} 2}\right|$ where $\Phi_{\mathrm{K} 1}$ (at angle $\angle_{\mathrm{K} 1}$ ) and $\Phi_{\mathrm{K} 2}$ (at angle $\angle_{\mathrm{K} 2}$ ) are the reported astigmatic powers. We used $\angle_{\mathrm{K} 1}$ for $\angle_{\mathrm{Cyl}}$. The anterior and posterior radii of curvature ( $r_{a}$ and $r_{p}$ respectively) were obtained from the reported values. The central thickness $(C C T)$ was the reported thickness at the corneal apex.

For all OCT biometry calculations, we obtained the anterior and posterior radii of curvature over a $5 \mathrm{~mm}$ diameter zone and calculated the central corneal thickness at the vertex of the cornea. ${ }^{23} \mathrm{We}$ used these measurements to calculate the thick lens refractive power, $\Phi_{\mathrm{EFL}}=$ $\left(n_{c}-n_{a}\right) / r_{a}+\left(n_{q}-n_{c}\right) /\left(r_{a} * r_{p}{ }^{*} n_{c}\right)$. To calculate the back vertex corrected refractive power, we used the formula: $\Phi_{B F L}=\left(n_{c} * r_{a} * \Phi_{\mathrm{EFL}}\right) /\left(n_{c} * r_{a}-C C T *\left(n_{c}-n_{a}\right)\right)$. We used the Gullstrand values found within the literature for phase indices of refraction for cornea $\left(n_{c}\right)$, air $\left(n_{a}\right)$, and aqueous $\left(n_{q}\right)$ where $n_{c}=1.376, n_{a}=1.000$, and $n_{q}=1.336 .5,28 \mathrm{We}$ used $\Phi_{\mathrm{BFL}}$ as $\Phi_{\mathrm{K}}$ to compare OCT to topography, Scheimpflug photography and manifest refraction ${ }^{31}$.

We extended the above methods to account for astigmatic refractive power, $\Phi_{C y l}$, and permit multi-surface corneal topography. Following the above segmentation process for each volume, we fit each resultant surface to a $5^{\text {th }}$ order Zernike polynomial. We generated a local thickness map (pachymetry) between the fit polynomial surfaces with the thickness 
taken normal to the anterior surface. In addition, from these polynomials we analytically calculated a meridional curvature map for each surface as outlined by the ANSI standard 'Corneal Topography Systems. ${ }^{32}$ Using the meridional curvature maps from the anterior and posterior corneal surfaces and the associated pachymetry map, we combined the data to generate a single refractive power topography map. Using this map, we calculated $\Phi_{\mathrm{K} 1}$ and $\Phi_{\mathrm{K} 2}$ along the principle axes $\angle_{\mathrm{K} 1}$ and $\angle_{\mathrm{K} 2}$ at the corneal vertex and numerically calculated an overall axial curvature map. We generated $\Phi_{\mathrm{Cyl}}$ from the formula $\left|\Phi_{\mathrm{K} 1}-\Phi_{\mathrm{K} 2}\right|$ and $\angle_{\mathrm{Cyl}}$ from $\angle_{\mathrm{K} 1}$.

\section{Corneal Biometry Comparisons and Statistics}

For all comparisons of analogous values, we utilized the mean of each set of repeated measures. While all devices imaged each eye in triplicate, in the event that a device had a single anomalous measurement (e.g. eye out of imaging modality field of view, blinking during acquisition) for a given eye, that measurement was excluded. If a device had multiple anomalous measurements for any given eye, that eye was excluded from the study.

Other than myopia or hyperopia, pre-operative subjects were otherwise normal. Placido-ring topography and Scheimpflug photography are accurate techniques to obtain corneal refractive power information in normal subjects. ${ }^{33-35}$ However, Placido-ring topography only provides information from the anterior surface of the cornea, thus we were only able to directly compare the anterior radius of curvature and the reported refractive power between all three devices. ${ }^{4}$ All subjects prior to surgery were used as controls to compare the measured anterior radius of curvature, $r_{a}$, and the spherical equivalent refractive power, $\Phi_{\mathrm{K}}$, of the three imaging devices. A sub-population was used to compare the astigmatic refractive power, $\Phi_{\mathrm{Cyl}}$, and angle, $\angle_{\mathrm{Cyl}}$. Subjects were included in this population if the measured corneal astigmatism (not manifest refraction astigmatism) among the three imaging devices had a median value of greater than or equal to 1 Diopter. Manifest refraction was not used as the reference as it would include contributions to astigmatism from the crystalline lens. We chose 1 Diopter cylinder as a conservative cutoff due to current toric intraocular lenses offering a minimum of 1.5 Diopter cylinder correction. ${ }^{36}$ Finally, we compared the pre- to post-operative change in spherical and astigmatic refractive powers, $\Delta \Phi_{\mathrm{K}}$ and $\Delta \Phi_{\text {Cyl }}$ respectively, as measured by the three imaging devices to the manifest refraction of subjects who returned $3+$ months post-surgery for evaluation and imaging. The change in astigmatic power was calculated by the method proposed by Jaffe and Clayman. ${ }^{37,} 38$ which considers astigmatism magnitude and direction.

Comparison of pre-operative analogous values $\left(r_{a}, \Phi_{\mathrm{K}}, \Phi_{\mathrm{K} 1}\right.$, and $\left.\angle_{\mathrm{K} 1}\right)$ between each imaging device was done in a pair-wise fashion via Bland-Altman plots. ${ }^{39} \mathrm{We}$ did not directly compare $\angle_{\mathrm{K} 2}$, between devices as it is orthogonal to $\angle_{\mathrm{K} 1}$ (i.e. it is $\pm 90^{\circ}$ from $\angle_{\mathrm{K} 1}$ ) and may be directly obtained from $\angle_{\mathrm{K} 1}$. The standard deviation of the repeated measures on each device was used to indicate the variability of intra-device measurements. Inter-device comparisons were not performed on $r_{p}$ or $C C T$ values for Placido-ring topography because it does not measure those values. Intra-device variability for $r_{p}$ and $C C T$ was calculated for Scheimpflug photography and OCT. Comparison of pre- to post-operative refractive change $\left(\Delta \Phi_{\mathrm{K}}\right.$ and $\Delta \Phi_{\mathrm{Cyl}}$ ) from each imaging device to manifest refraction was done in a pair-wise 
fashion via Bland-Altman plots. Paired t-tests with generalized estimating equations were used to identify any significant differences from zero (with $a=0.05$ ) for both inter-device comparisons and device-to-manifest comparisons. Generalized estimating equations were used to account for any possible correlation from including both eyes of subjects. Interdevice agreement and device-to-manifest agreement were determined by calculating pairwise intraclass correlation coefficients (ICC).

\section{Results}

\section{Normal Subjects: Spherical Corneal Refractive Power}

To validate the OCT derived corneal biometry, normal preoperative corneas were imaged and the derived measurements were compared against current clinical standards. Current clinical imaging devices such as topography or Scheimpflug photography are expected to correctly measure normal non-surgically altered corneas and serve as reference standards for comparison. A total of 38 subjects ( $15 \mathrm{M} / 23 \mathrm{~F}$ aged $22-55$ years old; $37 \mathrm{OD} / 35 \mathrm{OS}$ ) consented to enter the study. A total of 37 subjects ( $15 \mathrm{M} / 22 \mathrm{~F}$ from $22-55$ years old; 36 OD/34 OS) subjects completed the pre-operative imaging session. One subject did not successfully complete the preoperative examination for entry due to improper image acquisition and was excluded from final analysis.

Inter-device pair-wise comparisons in this normal preoperative population are detailed in Table 1. For the anterior corneal radius of curvature, $r_{a}$, there was no statistically significant mean paired difference between DSOCT and the topography or Scheimpflug photography measurements. The mean paired difference between topography and Scheimpflug photography was statistically significant. For the spherical equivalent refractive power, $\Phi_{\mathrm{K}}$, there was no statistically significant mean paired difference between DSOCT and Scheimpflug photography. The mean paired differences between topography and the other two modalities were both statistically significant. However, the mean paired differences between all three devices were below $0.25 \mathrm{D}$, the conventional resolution for spectacle prescriptions.

\section{Normal Subjects: Astigmatic Corneal Refractive Power}

From the above normal preoperative population, a sub-population of 21 subjects $(10 \mathrm{M} / 11 \mathrm{~F}$ aged 22-55 years old; $18 \mathrm{OD} / 14 \mathrm{OS}$ ) with astigmatism of greater than 1 Diopter was selected to compare measured major and minor axis corneal powers and axis. Inter-device comparisons are detailed in Table 2 showing the mean and standard deviation of pair-wise differences between devices for major and minor axis corneal power and major axis angle. We did not include the minor axis angle as it is, by definition, orthogonal to the major axis. ${ }^{32}$

For the orthogonal astigmatic powers $\left(\Phi_{\mathrm{K} 1}\right.$ and $\left.\Phi_{\mathrm{K} 2}\right)$, there were no statistically significant mean paired differences between any of the devices. For the major axis angle, $\angle_{\mathrm{K} 1}$, the mean paired difference between topography and DSOCT was not statistically significant. However, the $\angle_{\mathrm{K} 1}$ mean paired differences between Scheimpflug photography and the other two modalities were both statistically significant. 


\section{Normal Subjects: Intra-Device Repeatability}

To evaluate variability, intra-device repeatability was found by taking the population-mean of the standard deviation of repeated measurements on each eye. For the anterior radius of curvature, $r_{a}$, DSOCT repeatability (Table $3, \sigma^{-}= \pm 0.026 \mathrm{~mm}$ ) compared favorably to topography $\left(\sigma^{-}= \pm 0.033 \mathrm{~mm}\right)$ and Scheimpflug photography $\left(\sigma^{-}= \pm 0.023 \mathrm{~mm}\right)$ with a mean repeatability between both devices. Scheimpflug photography and OCT are both tomographic imaging techniques, thus providing depth information. This information was used on both devices to generate measurements for posterior radius of curvature, $r_{p}$, and central corneal thickness, $C C T$. For both $r_{p}$ and CCT, we found DSOCT $\left(r_{p} \sigma^{-}= \pm 0.012 \mathrm{~mm}\right.$, $\left.C C T \sigma^{-}= \pm 1.5 \mu \mathrm{m}\right)$ to have less variability than Scheimpflug photography $\left(r_{p} \sigma^{-}= \pm 0.042 \mathrm{~mm}\right.$, $\left.C C T \sigma^{-}= \pm 4.6 \mu \mathrm{m}\right)$. All three devices use separate methods to calculate spherical equivalent refractive power $\Phi_{\mathrm{K}}$ : topography utilizes solely $r_{a}{ }^{32}$, Pentacam utilizes the EKR formula 29 which includes $r_{a}$ and $r_{p}$, and DSOCT utilizes $r_{a}, r_{p}$, and $C C T$. We found that for $\Phi_{\mathrm{K}}$, DSOCT $(\sigma= \pm 0.17 \mathrm{D})$ was as repeatable as or better than Scheimpflug photography $\left(\sigma^{-}=\right.$ $\pm 0.17 \mathrm{D})$ or topography $\left(\sigma^{-}= \pm 0.19 \mathrm{D}\right)$. To evaluate $r_{a}$ and $\Phi_{\mathrm{K}}$ accuracy, we took the pair-wise differences between all three devices. For $r_{a}$, we found no statistical significance $(p>0.05)$ in the difference between DSOCT to either topography or Scheimpflug photography. We did find a statistically significant difference $(p=0.043)$ between Scheimpflug photography and topography. For $\Phi_{\mathrm{K}}$, only the difference between DSOCT and Scheimpflug photography was statistically insignificant $(p=0.296)$. The mean paired difference between topography and both DSOCT and Scheimpflug photography was statistically significant $(p<0.05)$. While there were statistical differences, the absolute mean paired difference between any two pair of devices was less than $0.25 \mathrm{D}$.

For $\Phi_{\mathrm{K} 1}$, Scheimpflug photography was the most repeatable at $\sigma= \pm 0.18 \mathrm{D}$ followed by topography $(\sigma= \pm 0.23 \mathrm{D})$ and DSOCT $(\sigma= \pm 0.26 \mathrm{D})$. For the minor axis, $\Phi_{\mathrm{K} 2}$, Scheimpflug photography was again the least variable $\left(\sigma^{-}= \pm 0.15 \mathrm{D}\right)$ followed by DSOCT $\left(\sigma^{-}= \pm 0.22 \mathrm{D}\right)$ then topography $\left(\sigma^{-}= \pm 0.26 \mathrm{D}\right)$. Finally, for $\angle_{\mathrm{K} 1}$ Scheimpflug photography saw the least variability $\left(\sigma^{-}= \pm 4.4^{\circ}\right)$ followed by topography $\left(\left(\sigma^{-}= \pm 7.6^{\circ}\right)\right.$ then DSOCT $\left(\sigma^{-}= \pm 11.9^{\circ}\right)$. While this implementation of DSOCT was somewhat more variable than topography or Scheimpflug photography for these astigmatic measurements, statistically the inter-device pair-wise differences (for both $\Phi_{\mathrm{K} 1}$ and $\Phi_{\mathrm{K} 2}$ ) between all three devices were not significant $(p>0.05)$. However, the $\angle_{\mathrm{K} 1}$ difference between Scheimpflug photography and both topography $(p=0.020)$ and DSOCT $(p=0.038)$ was statistically significant. The $\angle_{\mathrm{K} 1}$ between topography and DSOCT was not significant $(p=0.143)$.

\section{After LASIK: Measured Refractive Change Due to LASIK}

Of the 37 subjects that completed the pre-operative protocol, 27 subjects returned for postoperative imaging at least 3 months after their LASIK procedure. Of these 27 (26 OD/24 OS) subjects who returned, 5 were excluded from the final population: 3 subjects had improper image acquisitions and 2 did not complete the imaging protocol. In addition, one subject completed the protocol with one eye but not the other due to improper image acquisitions. A total of 22 subjects ( $8 \mathrm{M} / 14 \mathrm{~F}$ from 22-53 years old; $21 \mathrm{OD} / 18 \mathrm{OS}$ ) successfully completed both the pre- and post- operative imaging protocols. For these individuals, the change in vertexed manifest refraction pre to post LASIK served as a 
clinical reference. Because the change in refraction resulted from excimer laser ablation of the anterior cornea, each of the three imaging modalities should show a corresponding change.

Table 5 provides the pre to post LASIK comparison between vertexed change in clinical refraction (MRx) to the change measured by each of the three imaging modalities. The mean difference between the reference vertexed change in spherical equivalent MRx and measured topographic change was $-0.50 \mathrm{D}$ with a $95 \%$ confidence interval (CI) of $-0.27 \mathrm{D}$ to $-0.74 \mathrm{D}$; the mean difference between the reference and measured Scheimpflug photography change was $0.06 \mathrm{D}$ with a $95 \% \mathrm{CI}$ of $-0.25 \mathrm{D}$ to $0.37 \mathrm{D}$; and the mean difference between the reference and measured OCT change was $0.13 \mathrm{D}$ with a $95 \% \mathrm{CI}$ of $-0.10 \mathrm{D}$ to $0.36 \mathrm{D}$. Other comparisons for the individual patient data summarized by Table 5 are displayed in Fig. 3 and Fig. 4.

For change in spherical equivalent refractive power, $\Delta \Phi_{\mathrm{K}}$, the mean paired difference between the reference manifest refraction and topography was statistically significant. The mean paired differences between the reference manifest refraction and the other two imaging modalities (Scheimpflug photography and OCT) were not statistically significant. For surgically induced change in astigmatic power, $\Delta \Phi_{\mathrm{Cyl}}$, the mean paired differences between the reference manifest refraction and all three imaging modalities were statistically significant.

\section{Discussion}

In this work, we have shown that distributed-scanning motion-corrected OCT (DSOCT) can measure the actual corneal refractive power change due to LASIK, a measurement which to date has challenged the clinical standard of keratometry and similar measures such as topography based SimK. Using the change in manifest refraction due to LASIK as a reference, the OCT measured change was found to not differ significantly from this reference. Though a meaningful effect may be missed due to the post-operative sample size, this is unlikely given that the upper and lower limits of the $95 \%$ confidence interval for the mean difference between OCT and the reference $(-0.10 \mathrm{D}$ to $0.36 \mathrm{D})$ are similar to the \pm $0.25 \mathrm{D}$ resolution of clinical refraction. Examining the absolute difference from the reference, DSOCT also had the smallest mean difference and variability compared to the reference of the three imaging modalities. DSOCT's ability to measure the refractive power of the post LASIK cornea represents an important clinical advance. With the ability to measure the post LASIK cornea, performing nearly inevitable subsequent cataract surgery and selecting an appropriately powered intraocular lens for this growing population could become as routine and reliable as it is currently for those who have not had LASIK.

Further, we have also shown that DSOCT is capable of measuring astigmatic change with comparable accuracy to clinical standards. This is important because of the advent of astigmatism correcting intraocular lenses and other surgical procedures for correcting this higher order refractive error. In the normal cornea, DSOCT measured both the magnitude and direction of the astigmatism without a significant difference from the clinical standards. In the post LASIK cornea, the magnitude of surgically induced astigmatism measurements 
with topography and DSOCT were both found to be statistically different from the reference measurement. However, despite the statistical difference, the mean difference between OCT and the reference measurement $(0.07 \mathrm{D})$ would not be considered clinically significant given that the resolution of a clinical refraction is $\pm 0.25 \mathrm{D}$.

Spherical errors such as myopia and hyperopia are two of the largest contributors to refractive error ${ }^{40}$ and previous studies utilizing OCT to estimate corneal refractive error have only explored this primary influence. ${ }^{22,41}$ An early attempt that combined Placido-ring topography and time- domain OCT (TDOCT) ${ }^{41}$ found that the addition of the posterior surface information more closely matched the change in spherical equivalent manifest refraction due to refractive surgery compared to including only the information from the anterior surface. While the bias was reduced using both surfaces, the difference was still statistically significant. A later study from the same group, ${ }^{22}$ utilized a higher speed SDOCT system and analyzed the repeatability of spherical equivalent refractive power measurements. However, in this study they did not explore the measured change in refractive power due to surgery. In addition to spherical error, many individuals often have additional refractive errors due to other aberrations, such as astigmatism ${ }^{40}$ which neither of these earlier works addressed.

While a specialized scan pattern was used in our study to allow OCT to mitigate corrupting motion artifacts, this scan pattern was a software change that could readily be adopted by other OCT systems. Indeed, our entire process of scan acquisition and clinical measurement derivation is built on a current generation clinical SDOCT system. We would expect that other current generation clinical SDOCT could similarly utilize our developments via software modification of their systems. Because SDOCT currently has a wide installation base in the ophthalmic community, the developments in this work to allow measurement of the post LASIK cornea could potentially be widely available to the eye care community.

OCT compared well to manifest refraction with respect to the other imaging devices, but the difference between OCT and the reference was not zero. These differences are most likely due to residual effects of higher frequency patient motion and minor segmentation errors due to SDOCT falloff creating low contrast at the corneal anterior surface, ${ }^{23}$ both of which are hardware limitations. Given the scan pattern used for this study, improved imaging speed would potentially allow for reduction of higher frequency motion artifacts which would correspond to a reduction in variability for both spherical equivalent and astigmatic refractive power measurements. Alternatively, one could increase the density and total number of meridians utilized in the distributed scanning OCT scan pattern utilizing the improved scan speed. The $\Phi_{\mathrm{K} 1}$ and $\angle_{\mathrm{K} 1}$ ICC between Placido-ring topography and Scheimpflug photography was better than the ICC between DSOCT and either respective modality. This is likely because topography and Scheimpflug photography sampled more meridians (angles) of the cornea. In the next implementation of DSOCT, we expect to reduce the variability of astigmatic magnitude and angle measurements by increasing the total number of sampled meridians. With regards to SDOCT falloff, the clinical system used had a falloff of $\sim 2 \mathrm{~mm} .{ }^{23}$ Given that a normal cornea is $\sim 0.55 \mathrm{~mm}$ in central thickness and the total depth from corneal anterior surface apex to the periphery of the posterior surface, the margin of error for capturing the entire corneal profile in an image decreases as the scan 
width increases. For our study, the corneal periphery was $\pm 3 \mathrm{~mm}$ and assuming a posterior radius of curvature of $6.5 \mathrm{~mm}$, it can be shown a corneal profile occupies $\sim 1.3 \mathrm{~mm}$ of total depth providing only $\sim \pm 0.35 \mathrm{~mm}$ margin of error. For in vivo OCT imaging, the anterior corneal surface exhibits higher contrast than posterior surface with respect to the corneal stroma. This effect is most likely due to the large refractive index mismatch at the air-cornea interface at the anterior surface versus much smaller mismatch at the posterior surface interface. Because of this difference in contrast and the difference in SNR due to falloff within an OCT image, we placed the posterior surface of the cornea closer to $0 \mathrm{~mm}$ in depth and the anterior surface closer to $2 \mathrm{~mm}$ in depth. ${ }^{23}$ This is analogous to what is done in EDI retinal imaging. ${ }^{42}$ While this technique improved posterior surface segmentation and repeatability, ${ }^{23,} 24$ anterior surface contrast was decreased, which may have introduced minor segmentation variability between volumes. Next generation, high-speed swept-source OCT systems offer the potential to further reduce motion artifacts and limit the effects of falloff. 30,43

Manifest refraction may have also contributed to the variability between itself and the imaging devices. MRx is a subjective measurement requiring feedback from the individual patient stating when an image appears the most readable to them. Because of this, one individual may prefer an image that is optically sharper than another individual, thus introducing variability not accountable by independent, objective measurement techniques.

Additional variability that may be introduced by accommodation in younger patients can be mitigated by using cycloplegic refractions. However, as a patient specific measurement technique, MRx has been shown to have improved refractive surgery outcomes over device mediated measurements such as an aberrometer. ${ }^{44}$ While we could have used an aberrometer to measure the change in refractive error and potentially observed less pair-wise difference variability, we instead chose to use the clinical standard of care - MRx.

Corneal power is one variable used in modern intraocular lens selection formulas and is the one most directly impacted by laser refractive surgery. We have shown in this work that DSOCT importantly measures the corneal power change from these refractive surgeries, but we do not at this time recommend a direct replacement of keratometry values with DSOCT measurements in this population. Modern intraocular lens formulas are not purely theoretical formulas and have been empirically optimized over time. For example, keratometry values today serve not only to describe corneal refractive power but also as an anatomic measure to estimate the final intraocular lens position. ${ }^{6}$ Exploring these non-optical relationships in current formulas is necessary prior to directly using these improved corneal measurements for the post laser refractive surgery population.

In conclusion, motion corrected OCT more accurately measures the change in refractive power due to surgery than current clinical devices. By offering accurate corneal refractive measurements in normal and surgically modified subjects, OCT could replace current clinical standards for determining corneal refractive power.

\section{Acknowledgments}

Financial Support: This study was supported with funding from the Wallace H. Coulter Translational Partnership Award (Miami, FL), Research to Prevent Blindness (New York, NY), NIH R21 EY020001 (Bethesda, MD), and 
NIH K23 EY021522 (Bethesda, MD). These funding organizations had no role in the design or conduct of this research.

We would like to acknowledge Stephanie Chiu and Francesco LaRocca (Duke University) for their assistance with automated segmentation. We would also like to thank Dr. Alan Carlson, Dr. Terry Kim, Heidi Campbell, Ana Garcia-Turner, and Maria Perveiler for their assistance with subject enrollment.

\section{References and Notes}

1. Vision Research Needs, Gaps, and Opportunities. National Eye Institute; 2012.

2. Preferred Practice Pattern: Cataract in the Adult Eye. American Academy of Ophthalmology; 2011.

3. Seitz B, Langenbucher A, Nguyen NX, et al. Underestimation of intraocular lens power for cataract surgery after myopic photorefractive keratectomy. Ophthalmology. 1999; 106(4):693-702. [PubMed: 10201589]

4. Olsen T. On the calculation of power from curvature of the cornea. Brit J Ophthalmol. 1986; 70(2): 152-4. [PubMed: 3947615]

5. Schwiegerling, J. Field Guide to Visual and Ophthalmic Optics. Washington: SPIE Press; 2004.

6. Aramberri J. Intraocular lens power calculation after corneal refractive surgery: Double-K method. J Cataract Refr Surg. 2003; 29(11):2063-8.

7. Wang L, Booth MA, Koch DD. Comparison of intraocular lens power calculation methods in eyes that have undergone LASIK. Ophthalmology. 2004; 111(10):1825-31. [PubMed: 15465542]

8. Feiz V, Moshirfar M, Mannis MJ, et al. Nomogram-Based Intraocular Lens Power Adjustment after Myopic Photorefractive Keratectomy and LASIK: A New Approach. Ophthalmology. 2005; 112(8): 1381-7. [PubMed: 16061093]

9. Latkany RA, Chokshi AR, Speaker MG, et al. Intraocular lens calculations after refractive surgery. J Cataract Refr Surg. 2005; 31(3):562-70.

10. Masket S, Masket SE. Simple regression formula for intraocular lens power adjustment in eyes requiring cataract surgery after excimer laser photoablation. J Cataract Refr Surg. 2006; 32(3): 430-4.

11. Savini G, Barboni P, Zanini M. Intraocular Lens Power Calculation after Myopic Refractive Surgery: Theoretical Comparison of Different Methods. Ophthalmology. 2006; 113(8):1271-82. [PubMed: 16769117]

12. Borasio E, Stevens J, Smith GT. Estimation of true corneal power after keratorefractive surgery in eyes requiring cataract surgery: BESSt formula. J Cataract Refr Surg. 2006; 32(12):2004-14.

13. Shammas HJ, Shammas MC. No-history method of intraocular lens power calculation for cataract surgery after myopic laser in situ keratomileusis. J Cataract Refr Surg. 2007; 33(1):31-6.

14. Haigis W. Intraocular lens calculation after refractive surgery for myopia: Haigis-L formula. J Cataract Refr Surg. 2008; 34(10):1658-63.

15. Huang D, Swanson EA, Lin CP, et al. Optical Coherence Tomography. Science. 1991; 254(5035): 1178-81. [PubMed: 1957169]

16. Swanson EA, Izatt JA, Hee MR, et al. In vivo retinal imaging by optical coherence tomography. Opt Lett. 1993; 18(21):1864-6. [PubMed: 19829430]

17. Izatt JA, Hee MR, Swanson EA, et al. Micrometer-Scale Resolution Imaging of the Anterior Eye In Vivo With Optical Coherence Tomography. Arch Ophthalmol. 1994; 112(12):1584-9. [PubMed: 7993214]

18. Fercher AF, Hitzenberger CK, Kamp G, El-Zaiat SY. Measurement of intraocular distances by backscattering spectral interferometry. Opt Commun. 1995; 117(1-2):43-8.

19. Drexler W, Baumgartner A, Findl O, et al. Submicrometer precision biometry of the anterior segment of the human eye. Invest Ophthalmol Vis Sci. 1997; 38(7):1304-13. [PubMed: 9191593]

20. Wojtkowski M, Leitgeb R, Kowalczyk A, et al. In vivo human retinal imaging by Fourier domain optical coherence tomography. J Biomed Opt. 2002; 7(3):457-63. [PubMed: 12175297]

21. Sarunic MV, Asrani S, Izatt JA. Imaging the Ocular Anterior Segment With Real-Time, FullRange Fourier-Domain Optical Coherence Tomography. Arch Ophthalmol. 2008; 126(4):537-42. [PubMed: 18413525] 
22. Tang M, Chen A, Li Y, Huang D. Corneal power measurement with Fourier-domain optical coherence tomography. J Cataract Refr Surg. 2010; 36(12):2115-22.

23. McNabb RP, LaRocca F, Farsiu S, et al. Distributed scanning volumetric SDOCT for motion corrected corneal biometry. Biomed Opt Express. 2012; 3(9):2050-65. [PubMed: 23024900]

24. Kuo AN, McNabb RP, Zhao M, et al. Corneal biometry from volumetric SDOCT and comparison with existing clinical modalities. Biomed Opt Express. 2012; 3(6):1279-90. [PubMed: 22741075]

25. LaRocca F, Chiu SJ, McNabb RP, et al. Robust automatic segmentation of corneal layer boundaries in SDOCT images using graph theory and dynamic programming. Biomed Opt Express. 2011; 2(6):1524-38. [PubMed: 21698016]

26. Zhao M, Kuo AN, Izatt JA. 3D refraction correction and extraction of clinical parameters from spectral domain optical coherence tomography of the cornea. Opt Express. 2010; 18(9):8923-36. [PubMed: 20588737]

27. McNabb RP, Kuo AN, Izatt JA. Quantitative single and multi-surface clinical corneal topography utilizing optical coherence tomography. Opt Lett. 2013; 38(8):1212-4. [PubMed: 23595434]

28. Atchison, DA.; Smith, G. Optics of the human eye. Butterworth-Heinemann; 2000.

29. Holladay JT, Hill WE, Steinmueller A. Corneal Power Measurements Using Scheimpflug Imaging in Eyes with Prior Corneal Refractive Surgery. J Refract Surg. 2009; 25(10):862-8. [PubMed: 19835326]

30. Dhalla A-H, Nankivil D, Bustamante T, et al. Simultaneous swept source optical coherence tomography of the anterior segment and retina using coherence revival. Opt Lett. 2012; 37(11): 1883-5. [PubMed: 22660061]

31. Norrby NE. Unfortunate discrepancies. J Cataract Refr Surg. 1998; 24(4):433-4.

32. ANSI. Corneal Topography Systems. 2007; Z80:23-2007.

33. Hannush S, Crawford S, Waring GI, et al. Reproducibility of normal corneal power measurements with a keratometer, photokeratoscope, and video imaging system. Arch Ophthalmol. 1990; 108(4): 539-44. [PubMed: 2322156]

34. Shankar H, Taranath D, Santhirathelagan CT, Pesudovs K. Anterior segment biometry with the Pentacam: Comprehensive assessment of repeatability of automated measurements. J Cataract Refr Surg. 2008; 34(1):103-13.

35. Shirayama M, Wang L, Weikert MP, Koch DD. Comparison of Corneal Powers Obtained from 4 Different Devices. Am J Ophthalmol. 2009; 148(4):528-35. e1. [PubMed: 19541287]

36. Alcon. AcrySofIQ Toric Astigmatism IOL. 2012

37. Jaffe N, Clayman $\mathrm{H}$. The pathophysiology of corneal astigmatism after cataract extraction. Trans Am Acad Ophthalmol Otolaryngol. 1975; 79(2):615-30.

38. Alpins NA, Goggin M. Practical astigmatism analysis for refractive outcomes in cataract and refractive surgery. Surv Ophthalmol. 2004; 49(1):109-22. [PubMed: 14711444]

39. Bland JM, Altman DG. Statistical methods for assessing agreement between two methods of clinical measurement. Lancet. 1986; 1(8476):307-10. [PubMed: 2868172]

40. Vitale S, Ellwein L, Cotch MF, et al. Prevalence of refractive error in the United States, 19992004. Arch Ophthalmol. 2008; 126(8):1111-9. [PubMed: 18695106]

41. Tang M, Li Y, Avila M, Huang D. Measuring total corneal power before and after laser in situ keratomileusis with high-speed optical coherence tomography. J Cataract Refr Surg. 2006; 32(11): 1843-50.

42. Spaide RF, Koizumi H, Pozonni MC. Enhanced Depth Imaging Spectral-Domain Optical Coherence Tomography. Am J Ophthalmol. 2008; 146(4):496-500. [PubMed: 18639219]

43. Grulkowski I, Liu JJ, Potsaid B, et al. Retinal, anterior segment and full eye imaging using ultrahigh speed swept source OCT with vertical-cavity surface emitting lasers. Biomed Opt Express. 2012; 3(11):2733-51. [PubMed: 23162712]

44. Reinstein DZ, Morral M, Gobbe M, Archer TJ. Accuracy of refractive outcomes in myopic and hyperopic laser in situ keratomileusis: Manifest versus aberrometric refraction. J Cataract Refr Surg. 2012; 38(11):1989-95. 


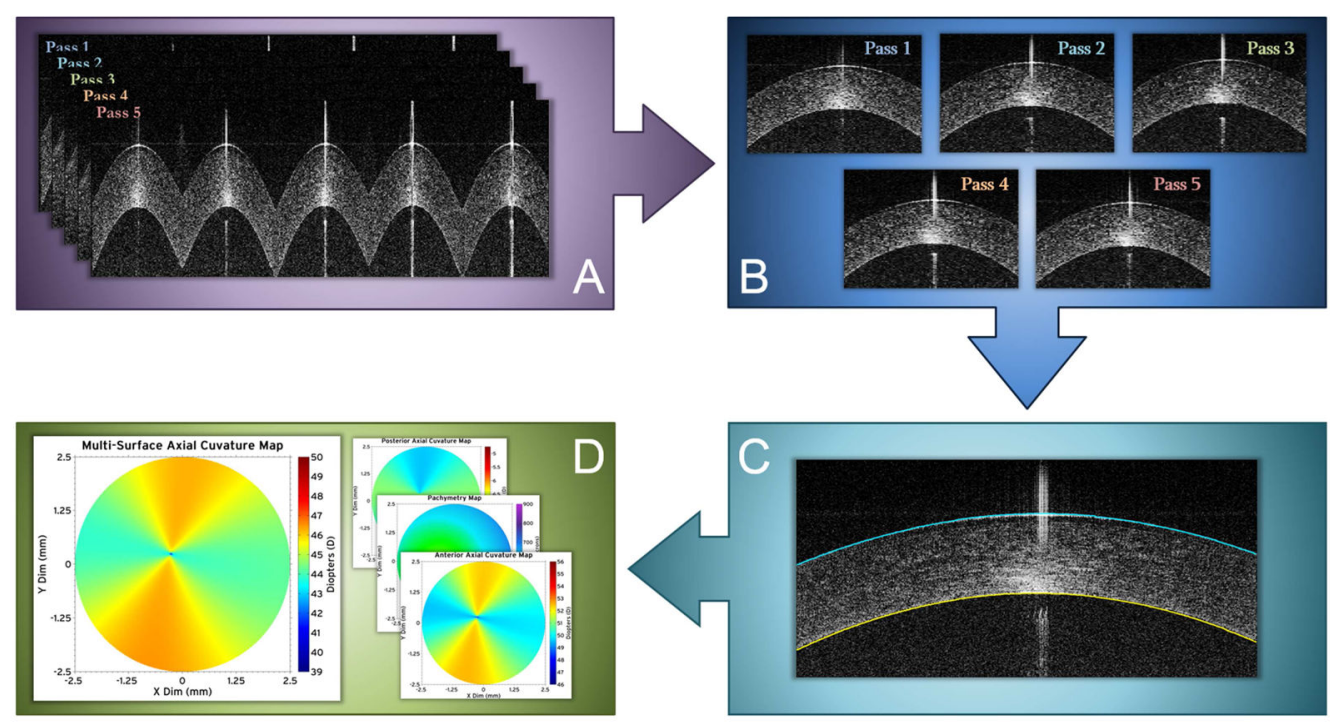

Fig. 1.

Process to extract clinically relevant measures from corneal OCT. (A) A distributed scan OCT volume is acquired of a cornea with a clinical OCT system. (B) Each distributed scanning OCT B-scan meridian is reconstructed from multiple sub-sampled B-scans along a given meridian to mitigate patient motion ${ }^{23}$. (C) Each reconstructed meridian is automatically segmented. The anterior corneal surface segmentation is depicted in cyan and the posterior surface segmentation in yellow. (D) The surface segmentations are corrected for distortions in the scanning optics and refraction within the cornea. The corrected segmentations are then used to generate corneal topographic maps for the anterior and posterior surfaces of the cornea and calculate local corneal thicknesses. These maps are then used to calculate overall corneal spherical and astigmatic refractive power. ${ }^{27}$ 


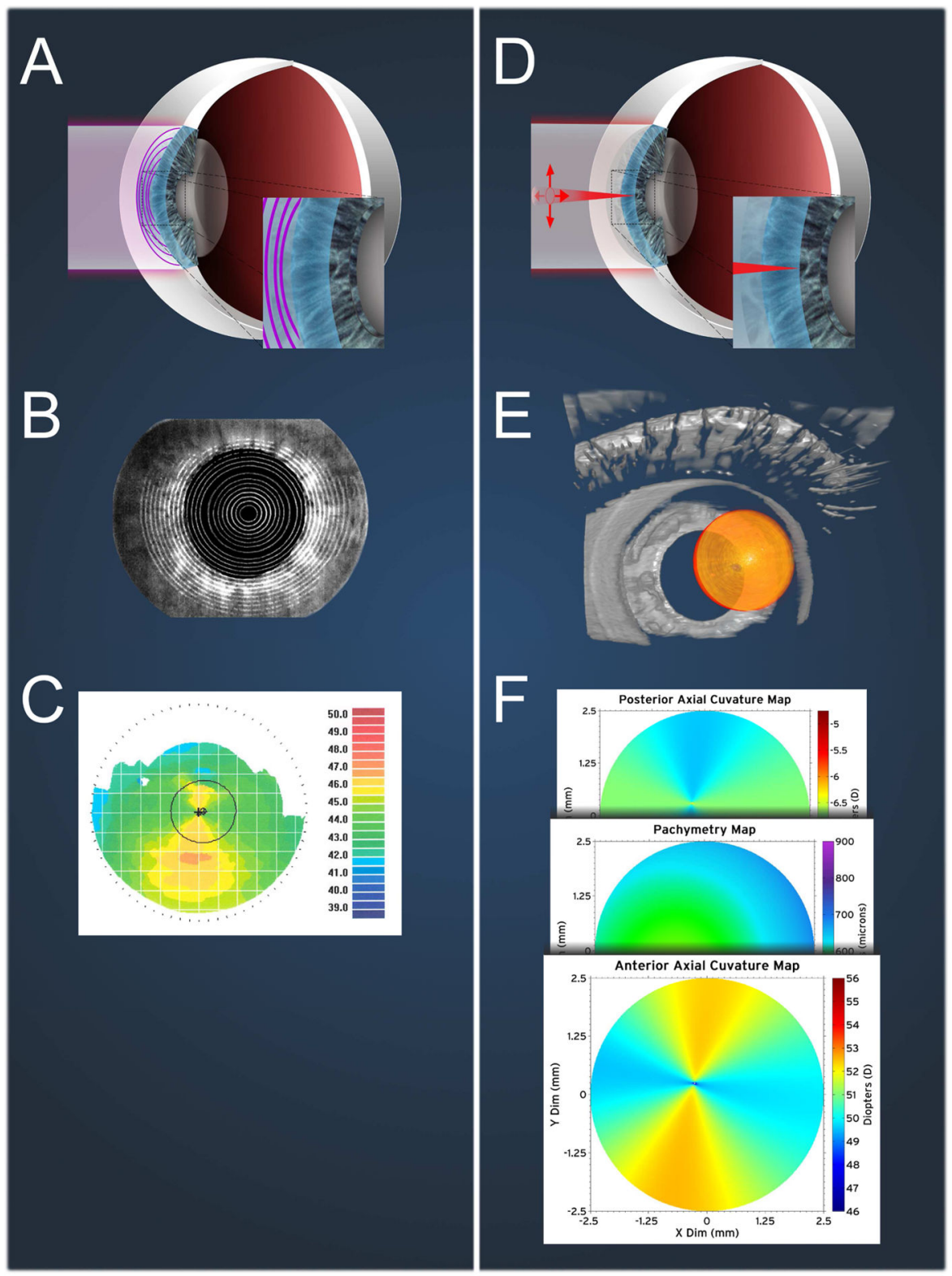

Fig. 2.

Comparison of Placido-ring corneal topography and OCT corneal topography (A) Placidoring topography projects concentric rings onto the anterior surface of the cornea (B) The shape of the imaged rings describe the curvature of the anterior surface $(\mathbf{C})$ By assuming certain characteristics of the corneal thickness and posterior surface a clinical topography map is generated from only the anterior surface (D) OCT utilizes a flying spot scanning technique where a focused beam of infrared light is scanned over the region where each spot corresponds to a single depth profile, or A-scan. As the spot is moved over the sample a volume may be constructed from sequential A-scans (E) Orange: OCT volume acquired from a clinical spectral domain OCT system corresponding to vertex of the cornea Grey: Volume from an experimental swept source OCT system ${ }^{30}$ providing geographic context for 
the spectral domain OCT volume (F) Maps created from OCT volume. Front: Topography map of the anterior corneal surface Middle: Local corneal thickness Back: Topography map of the posterior corneal surface 

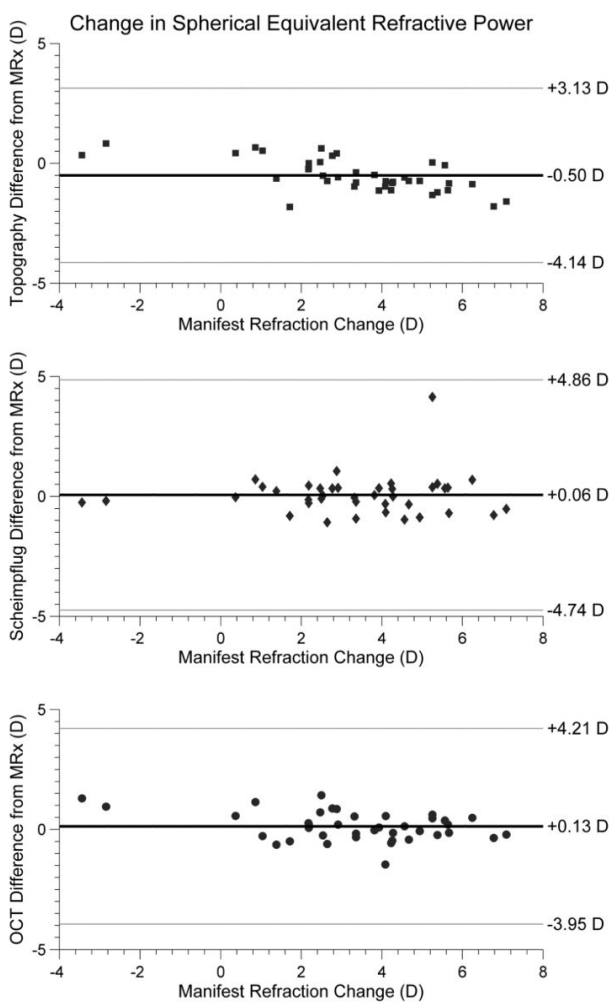

Fig. 3.

Comparison of measured change in corneal spherical equivalent refractive power due to LASIK. Top - Placido-ring Topography, Middle - Scheimpflug Photography, Bottom DSOCT. The plots show the reference manifest refractive change due to LASIK ( $\mathrm{x}$ axis) and difference from manifest refraction as measured by the imaging modalities (y axis). The scatter points represent measurements from individual eyes. The grey lines indicate \pm 1.96 standard deviations from the population mean (black line) for the corresponding modality. 

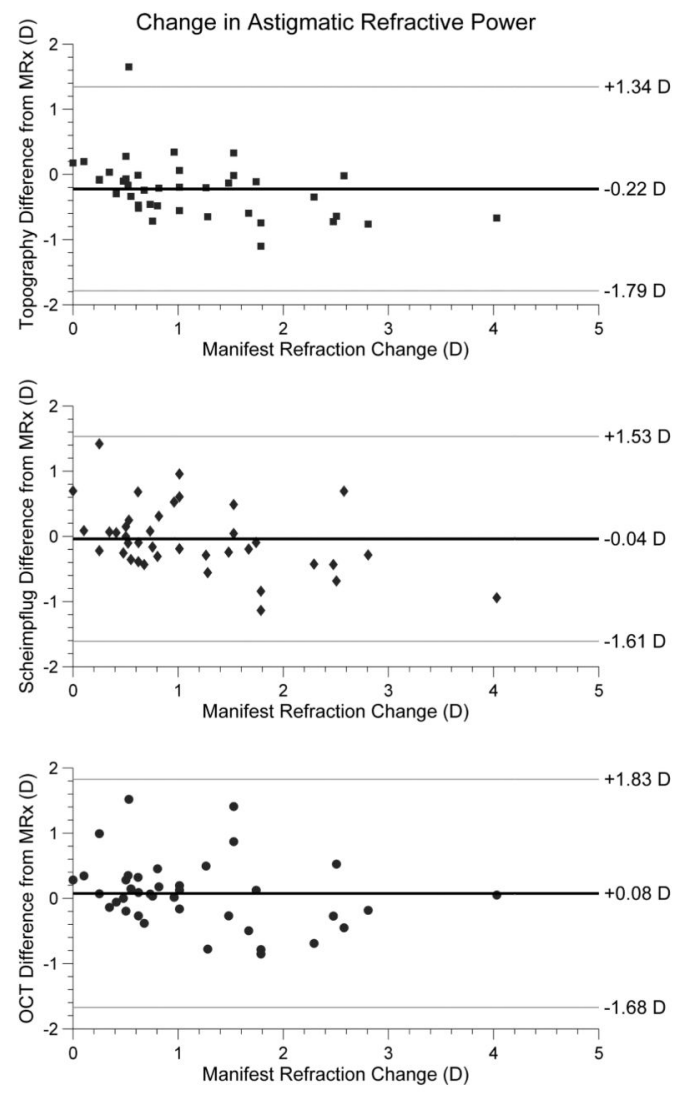

Fig. 4.

Comparison of measured change in corneal astigmatism due to LASIK. Top - Placido-ring Topography, Middle - Scheimpflug Photography, Bottom - DSOCT. The plots show the reference manifest refractive change due to LASIK ( $\mathrm{x}$ axis) and difference from manifest refraction as measured by the imaging modalities (y axis). The points represent measurements from individual eyes. The grey lines indicate \pm 1.96 standard deviations from the population mean (black line) for the corresponding modality. 


\section{Table 1}

Inter-device pair-wise comparison of $r_{a}$ (anterior corneal radius) and $\Phi_{\mathrm{K}}$ (corneal refractive power) for the preLASIK population. The mean and standard deviation of pair-wise differences between devices are reported. The $p$ values resulting from t-tests with generalized estimating equations and the intraclass correlations (ICC) with $95 \%$ confidence intervals of the ICC (in parentheses) are also reported. DSOCT refers to distributed scanning OCT.

\begin{tabular}{|c|c|c|c|}
\hline$\left(\mathbf{x}^{-}-\overline{\mathbf{y}}\right) \pm \sigma^{-}$ & Topography - DSOCT & DSOCT - Scheimpflug & Scheimpflug - Topography \\
\hline \multirow{2}{*}{$\boldsymbol{r}_{\boldsymbol{a}}(\mathrm{mm})$} & $-0.004 \pm 0.069$ & $-0.009 \pm 0.066$ & $0.012 \pm 0.056$ \\
& $p=0.675(0.96,0.94-0.98)$ & $p=0.406(0.96,0.94-0.98)$ & $p=0.043(0.97,0.96-0.98)$ \\
\hline \multirow{2}{*}{$\Phi_{\mathbf{K}}(\mathrm{D})$} & $0.15 \pm 0.42$ & $0.07 \pm 0.45$ & $-0.22 \pm 0.39$ \\
& $p=0.013(0.95,0.93-0.97)$ & $p=0.296(0.95,0.92-0.97)$ & $p=<0.001(0.95,0.92-0.97)$ \\
\hline
\end{tabular}




\section{Table 2}

Inter-device pair-wise comparison of orthogonal astigmatic power $\left(\Phi_{\mathrm{K} 1}, \Phi_{\mathrm{K} 2}\right)$ and major axis angle $\left(\angle_{\mathrm{K} 1}\right)$ for the pre-LASIK population with astigmatism greater than 1 Diopter. The mean and standard deviation of pairwise differences between devices are reported. The $p$ values resulting from t-tests with generalized estimating equations and the intraclass correlations (ICC) with 95\% confidence intervals (in parentheses) are also reported. DSOCT refers to distributed scanning OCT.

\begin{tabular}{|c|c|c|c|}
\hline$\left(\mathbf{x}^{-}-\overline{\mathbf{y}}\right) \pm \sigma^{-}$ & Topography - DSOCT & DSOCT - Scheimpflug & Scheimpflug - Topography \\
\hline \multirow{3}{*}{$\Phi_{\mathbf{K} 1}(\mathrm{D})$} & $0.11 \pm 0.57$ & $-0.05 \pm 0.63$ & $-0.07 \pm 0.48$ \\
& $p=0.302$ & $p=0.746$ & $p=0.428$ \\
& $I C C=0.91(0.82-0.95)$ & $I C C=0.89(0.79-0.94)$ & $I C C=0.94(0.87-0.97)$ \\
\hline \multirow{3}{*}{$\Phi_{\mathbf{K} 2}(\mathrm{D})$} & $-0.06 \pm 0.56$ & $0.10 \pm 0.54$ & $-0.04 \pm 0.43$ \\
& $p=0.605$ & $p=0.370$ & $p=0.583$ \\
& $I C C=0.92(0.85-0.96)$ & $I C C=0.92(0.85-0.96)$ & $I C C=0.95(0.90-0.98)$ \\
\hline \multirow{3}{*}{$\angle_{\mathbf{K} 1}\left({ }^{\circ}\right)$} & $-5.0 \pm 21.0$ & $8.9 \pm 25.2$ & $-3.9 \pm 9.6$ \\
& $p=0.143$ & $p=0.038$ & $p=0.020$ \\
& $I C C=0.86(0.74-0.93)$ & $I C C=0.79(0.62-0.89)$ & $I C C=0.96(0.91-0.98)$ \\
\hline
\end{tabular}


Table 3

Spherical equivalent intra-device population mean values and population mean variability for pre-operative population

\begin{tabular}{|c|c|c|c|}
\hline $\mathbf{x}\rceil \sigma^{-}$ & Placido-Ring Topography & Scheimpflug Photography & DSOCT \\
\hline $\boldsymbol{r}_{\boldsymbol{a}}(\mathrm{mm})$ & $7.692 \mid \pm 0.033$ & $7.705 \mid \pm 0.023$ & $7.696 \mid \pm 0.026$ \\
\hline $\boldsymbol{r}_{\boldsymbol{p}}(\mathrm{mm})$ & - & $6.391 \mid \pm 0.042$ & $6.545 \mid \pm 0.012$ \\
\hline $\boldsymbol{C C T}(\mu \mathrm{m})$ & - & $553 \mid \pm 4.6$ & $559 \mid \pm 1.5$ \\
\hline$\Phi_{\mathbf{K}}(\mathrm{D})$ & $43.93 \mid \pm 0.19$ & $43.71 \mid \pm 0.17$ & $43.78 \mid \pm 0.17$ \\
\hline
\end{tabular}


Table 4

Astigmatic intra-device population mean values and population mean variability for pre-operative population

\begin{tabular}{|c|c|c|c|}
\hline $\mathbf{x}\rceil \sigma^{-}$ & Placido-Ring Topography & Scheimpflug Photography & DSOCT \\
\hline$\Phi_{\mathbf{K} 1}(\mathrm{D})$ & $44.72 \mid \pm 0.23$ & $44.65 \mid \pm 0.18$ & $44.60 \mid \pm 0.26$ \\
\hline$\Phi_{\mathbf{K} 2}(\mathrm{D})$ & $43.04 \mid \pm 0.26$ & $43.01 \mid \pm 0.15$ & $43.1 \mid \pm 0.21$ \\
\hline$\left.\angle_{\mathbf{K} 1}{ }^{(}\right)$ & $91.8 \mid \pm 7.6$ & $88.0 \mid \pm 4.4$ & $96.9 \mid \pm 11.9$ \\
\hline
\end{tabular}




\section{Table 5}

Pair-wise differences and absolute pair-wise differences between pre to post LASIK changes in the reference manifest refraction change and the three imaging modalities (topography, Scheimpflug photography, and OCT). Comparisons are made for both changes in spherical equivalent ( $\triangle \Phi \mathrm{K}$ in white) and astigmatic powers $\left(\Delta \Phi_{\mathrm{Cyl}}\right.$ in grey). The $p$ values resulting from t-tests with generalized estimating equations and the intraclass correlations (ICC) with 95\% confidence intervals (in parentheses) are also reported. DSOCT refers to distributed scanning OCT.

\begin{tabular}{|c|c|c|c|}
\hline$\left(x^{-}-\bar{y}\right) \pm \sigma^{-}$ & MRx - Topography & MRx - Scheimpflug & MRx - DSOCT \\
\hline$\Delta \Phi_{\mathrm{K}}(\mathrm{D})$ & $\begin{array}{c}-0.50 \pm 0.68 \\
p=<0.001 \\
I C C=0.92(0.85-0.96)\end{array}$ & $\begin{array}{c}0.06 \pm 0.85 \\
p=0.714 \\
I C C=0.94(0.88-0.97)\end{array}$ & $\begin{array}{c}0.13 \pm 0.60 \\
p=0.216 \\
I C C=0.96(0.93-0.98)\end{array}$ \\
\hline$\left|\Delta \Phi_{\mathrm{K}}\right|(\mathrm{D})$ & $0.72 \pm 0.45$ & $0.53 \pm 0.66$ & $0.48 \pm 0.37$ \\
\hline$\Delta \Phi_{\text {Cyl }}(\mathrm{D})$ & $\begin{array}{c}-0.22 \pm 0.46 \\
p=0.003\end{array}$ & $\begin{array}{c}-0.04 \pm 0.52 \\
p=0.663\end{array}$ & $\begin{array}{c}0.07 \pm 0.53 \\
p=0.026\end{array}$ \\
\hline$\left|\Delta \Phi_{\text {Cyl }}\right|(\mathrm{D})$ & $0.60 \pm 0.51$ & $0.75 \pm 0.59$ & $0.49 \pm 0.37$ \\
\hline
\end{tabular}

